\title{
TUBERCULOUS PERICARDITIS
}

\author{
BY \\ H. L. HEIMANN AND S. BINDER \\ From the Johannesburg Hospital, South Africa \\ Received February 7, 1940
}

In Europe tuberculous pericarditis is uncommon as a primary clinical manifestation, even in the constrictive form that is suitable for operative treatment (White, 1937). Observation over fifteen years in the Non-European Hospital, Johannesburg, has shown that here it is not rare as a primary clinical entity. In this series of 31 cases some were of this type and some were only part of a generalized tuberculæmia ; there were none of the healed constrictive type.

The importance of the first type is that we have found it amenable to treatment. Unfortunately, Bantu patients are difficult to keep in hospital for long and almost impossible to follow up.

Some fifteen years ago a case was seen with œdema of the left arm and of the face, without albuminuria. There was pyrexia, but no classical signs of heart failure. The increased area of cardiac dullness suggested a pericardial effusion and the pear-shaped shadow with an obtuse cardio-hepatic angle was confirmed by radioscopy. Repeated pericardial punctures found no fluid. Autopsy showed that the pericardial sac was occupied by caseous material of great thickness, the myocardium being reduced to a thin sheet. Other somewhat similar cases were seen ; a single puncture only was done in some and fluid was absent. In 1938 a number of such cases were seen by both of us, differing only in that many of them had effusions, so that confirmation of the diagnosis by direct and biological methods could be made. Few of these later cases showed what had been considered the cardinal clinical sign, viz. œdema of the arm or face.

They were generally admitted as cases of congestive heart failure. There was an intermittent, remittent, or irregular fever. The shape of the heart could generally be outlined at the bedside accurately enough to differentiate it from the heart of beri-beri or from rheumatic or syphilitic heart disease with failure. Rheumatic pericarditis with effusion is very rare in these people (though the dry variety is not uncommon), so the finding of a large "pericardical-shaped heart" in a Bantu justifies a clinical diagnosis of tuberculous pericarditis, when rheumatic manifestations and evidence of mitral and aortic disease are absent.

The area of cardiac dullness sometimes attained huge proportions and in one case extended almost from axilla to axilla and yielded over a pint of fluid on 
aspiration. The heart sounds were usually muffled and murmurs were absent. In some cases a pericardial rub was heard. The pulse was generally rapid, 90-120, and a pulsus paradoxus was occasionally present. Extrasystoles and fibrillation were rare.

The signs in the lungs were generally those of congestion, and our series showed that the presence of pulmonary tuberculosis as a clinical entity was not necessary for the diagnosis of the condition. In the differential diagnosis the association of pulmonary tuberculosis and congestive heart failure should make one consider the question of tuberculous pericarditis. Pleural effusions were frequently found and were often bilateral.

Symptoms were vague, as might be expected in a primitive people, not able to describe their feelings accurately. In some cases there was precordial pain, and abdominal pain was common and was usually localized to the right hypochondrium, due to the acutely tender liver of recent rapid cardiac failure. The other symptoms were those common to cardiac failure.

Confirmation of the clinical diagnosis was often made at autopsy and in others by biological examination of the pericardial fluid. This fluid was sometimes serous, but most often hæmorrhagic. In several cases fluid could not be obtained and subsequent autopsy showed caseating material only.

The radiographic findings confirmed the cardiac enlargement and showed an obtuse hepato-cardiac angle. A typical illustration is given in Fig. 1. In

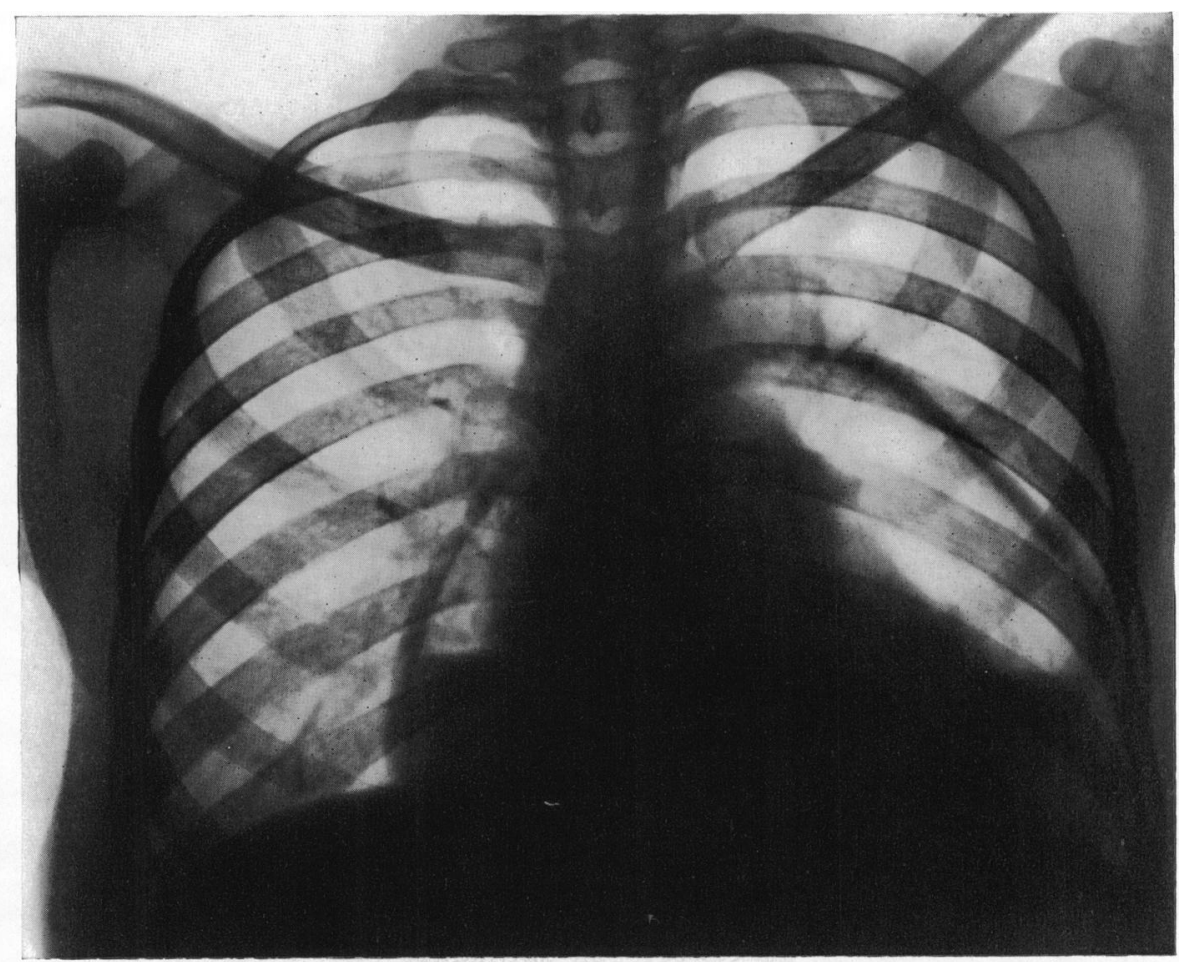

FIG. 1.-Routine teleradiogram showing the hydro-pneumo-pericardium. 
three cases kymographic tracings were obtained by the courtesy of Dr. Weinbren and Dr. Meyer. One after pneumopericardium is shown in Fig. 2. It was

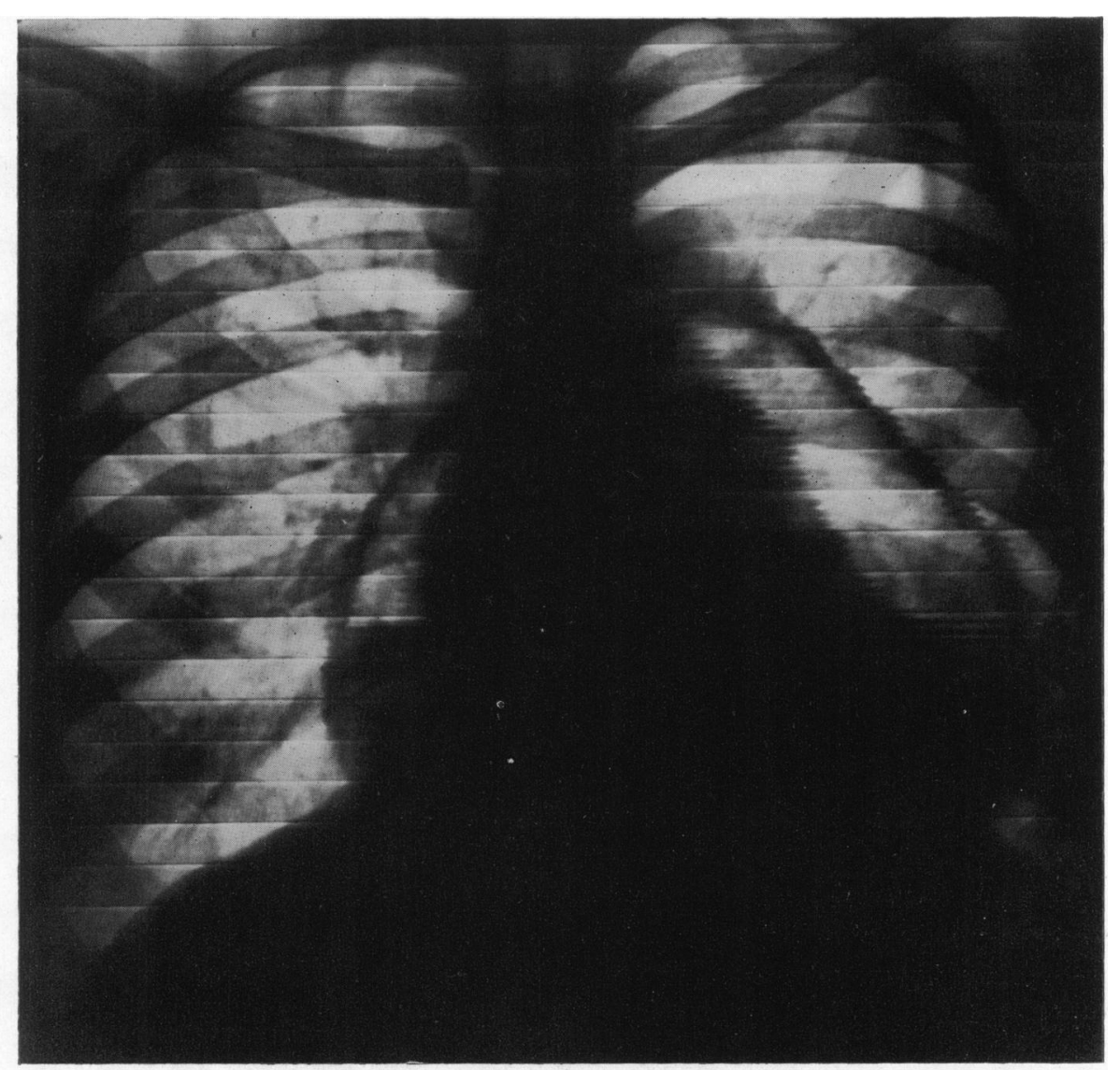

FIG. 2.-A kymogram taken with a moving grid in one second. The cardiac pulsations in the portion surrounded by air are well marked. Note that the peaks on either side of the pericardium are not opposite each other.

taken with a moving grid in one second. The long lines at the junction of air and fluid are due to splashing of the fluid. The cardiac pulsations in the portion surrounded by air are well marked, but below the level of the fluid they cannot be detected, either because of the density of the fluid or because the cardiac pulsation is damped down. In this case even if the cardiac pulsations were not damped down by the fluid, the density of the fluid would still have obscured them. The thickened pericardium itself shows transmitted pulsation. The kymogram of another case is shown in Fig. 3, where the pericardial effusion has completely damped down the pulsations of the heart itself.

In this series, dating 1925-1938, 31 cases were found in the records of the hospital, and of these 10 were seen in the first half of 1938 . The cases diagnosed clinically were those with the picture described above. The others were part 


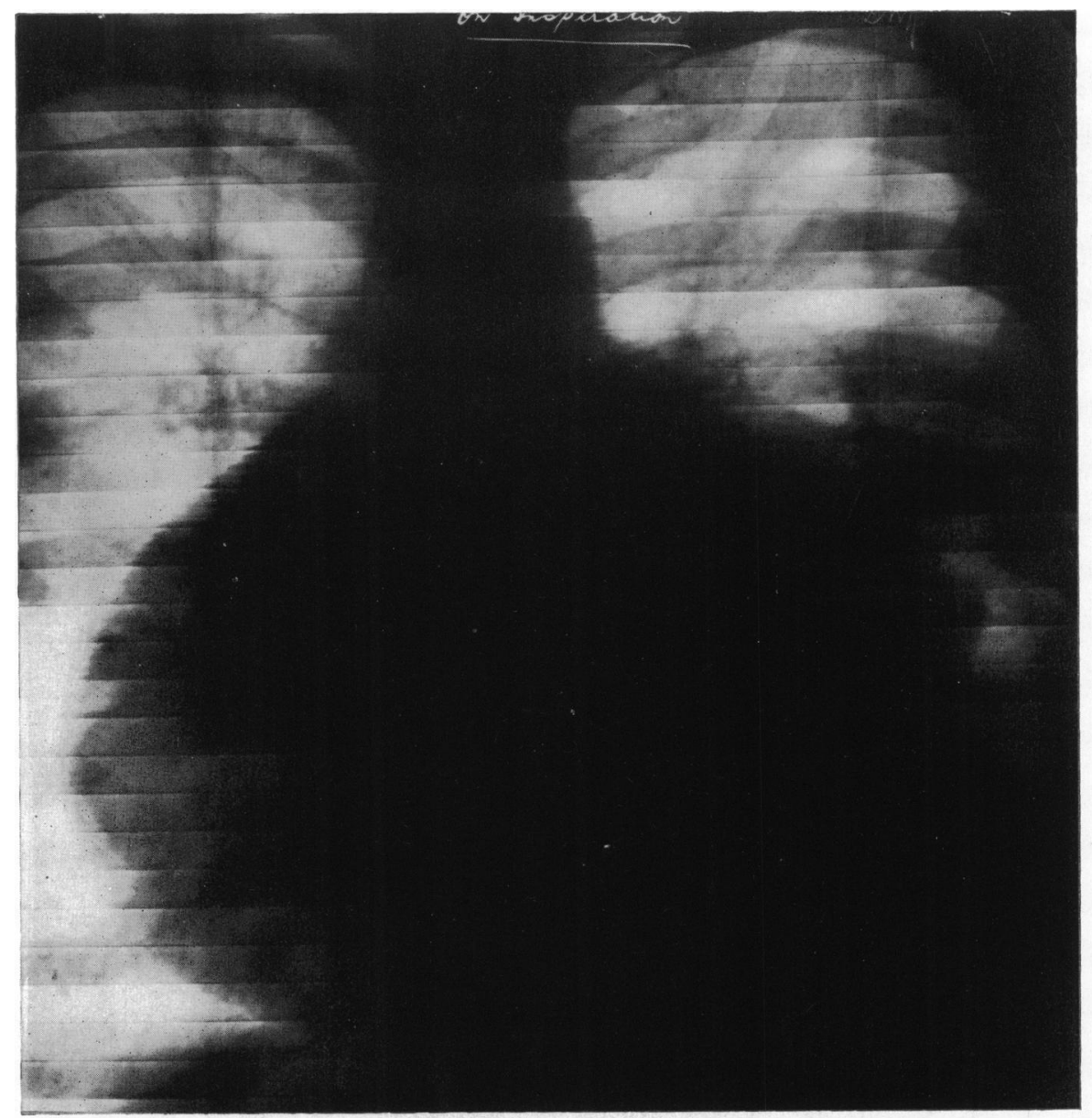

FIG. 3.-A kymogram taken in inspiration. Pulsations can be detected in the great vessels. The pericardial effusion has completely damped down the pulsations of the heart itself.

of a generalized tuberculosis and are included for the purposes of discussion as to the path of infection and pathological development of the condition. Some of the latter were of the adhesive type, but being part of a generalized tuberculæmia were not referred to the surgeons.

\section{DESCRIPTION OF CASES}

The nature of the condition and its progress have been a source of conjecture to some authors. In common with others, we believe that it begins acutely, then passes into a stage of effusion or caseation, and finally develops an adherent stage.

For the purposes of description our series is divided into three groups :

(1) Those with a normal area of cardiac dullness ;

(2) Those with a greatly enlarged area of cardiac dullness; and

(3) Those passing through various stages of the condition. 


\section{(1) Cases with a Normal Area of Cardial Dullness}

This group comprises 9 cases with an adherent pericarditis, as a result of which there was obliteration of the pericardial sac.

Sex and Age.-There were 6 males and 3 females. The age limits were between 15 and 76 years.

Histories.-These varied, particularly in regard to the patients' chief symptom. There was a cough in all cases, but only once an hæmoptysis. The other symptoms were chest pain, frequently over the precordium (4), ${ }^{*}$ breathlessness (2), œdema of the legs (2), and a generalized œdema, involving the face, arms, and legs (1) ; abdominal pain, usually localized to the right hypochondrium (4), diarrhœa with the passage of blood-stained stools (2), swellings situated over the sternum (2), and loss of weight (7).

Temperature and Pulse Rate.-Febrile charts were present in 6, the fever being either intermittent or remittent in character.

The pulse rate was increased in 8 ; auricular fibrillation was present in 1 , and this was the only irregularity. The respiratory rate was increased in 4 ; in 2 others occasional attacks of breathlessness occurred.

Clinical Signs. Cardiovascular.-Slight cardiac enlargement to the left was found in 2 ; in neither of these did the outline of the area of cardiac dullness suggest a pericarditis with effusion ; 1 had an increased area of cardiac dullness, which later, however, became normal. Normal heart sounds were found in 7. Bilateral pleural effusions were present in 2, and in 1 there was a left-sided effusion.

Abdominal.-Generalized abdominal tenderness was found in 1, and in 2 the abdomen had a doughy feel. Ascites was present in 5, the liver was enlarged and tender in 2, and irregular abdominal masses were present in 1 other.

Autopsy.-An adherent tuberculous pericarditis was present in all. Extrapericardial adhesions between the pericardium and surrounding organs were only found in 1 -in this instance between the pericardium and the chest wall. Slight cardiac enlargement was present in 3 . Tuberculous infiltration of the myocardium was found in 3, in 2 of which it was generalized, involving both auricles and ventricles, and in the other it was localized to the right auricle. Pulmonary tuberculosis was found in 5, including 1 with a commencing tuberculous broncho-pneumonia. In all the others odema and congestion of the lungs were present. The outstanding feature was that in all cases the root glands were tuberculous.

Generalized abdominal tuberculosis was present in 2. A tuberculous abscess of the chest was present in 1. Pulmonary infarcts were present in 1 case.

The diagnosis in this group is much more difficult than in the subsequent group. Very few cases in this group showed signs of cardiac failure. Pathologically the adhesions which formed were usually intra-pericardial and very rarely extra-pericardial.

* The numbers in brackets indicate the number of patients with this symptom. 
(2) Cases with a Greatly Enlarged Area of Cardiac Dullness

This group is made up of the 19 cases with either a serous, hæmorrhagic, or caseous exudate in the pericardial sac on the one hand, or those with loculation of the fluid due to adhesions between the visceral and parietal pericardial layers on the other hand. Loculation was proved, usually at autopsy, and indicated the start of the adherent stage.

Sex and Age.-There were 5 females and 14 males. The age incidence varied between 19 and 60 , the commonest age being between 20 and 40 years.

Histories. - The duration of illness varied from a week to three years, the commonest period being about three months. Cough was complained of in 18 cases ; one had an hæmoptysis and three others had at times coughed up blood-stained sputum ; in one of the latter, it was found that she had had pneumonia. In 7 there was a history of pain ( 5 precordial and 2 right-sided). Breathlessness on exertion was noted by 13 patients, and 10 complained of œdema of the legs.

Swelling of the abdomen was found in 3 and abdominal pain in 10, usually localized to the right hypochondrium. Loss of weight, very marked in some instances, occurred in 9 ; only 2 complained of night sweats.

Temperature and Pulse Rate.-Remittent or intermittent fever occurred in 14.

The pulse rate was raised in 17 , and pulsus paradoxus was found in 2 . The respiratory rate was increased in 16 cases.

Clinical Signs.- Edema of the legs was present in 7, and one of these had œdema of the face in addition. 13 had evidence, in some instances very marked, of wasting.

Cardiovascular.-The apex beat was palpable in 1 only. Gross enlargement of the area of cardiac dullness was found in 17. Pleural effusions obscured the cardiac outline in 2, and in some cases the area of dullness extended from axilla to axilla. The area of cardiac dullness was in most instances characteristically pear-shaped, with an obtuse cardio-hepatic angle. In all cases the heart sounds were normal, but generally muffled and distant. The types of pericardial effusions found will be dealt with under pathology.

Respiratory.-Signs indicating the presence of pulmonary tuberculosis were found in 5 , the commonest clinical signs being an impaired note at one or both apices, with moist sounds in the chest. Bilateral pleural effusions were present in 2, whilst 3 had right-sided effusions. In 2 a left-sided pleural effusion developed after aspiration of the pericardium.

Abdominal.-An enlarged and very tender liver was found in 10, and $3 \mathrm{had}$ ascites.

Pathology or Autopsy.-Autopsies were carried out in 16, and tuberculous pericarditis with effusion was present in all. In one just over four pints of effusion was present, whilst in others as little as 30-50 c.c. were found. In 7 the effusion was purely hæmorrhagic, in 5 caseous, and in 2 serous. Mixed types of effusion were present in 5 cases ; 3 hæmorrhagic and caseous ; 1 hæmorrhagic and fibrinous; and 1 caseous and serous. In 6 the effusion was found to be loculated by intra-pericardial adhesions. In 7 the heart was found to be small. Slight cardiac enlargement was found in 1, and 1 had a bread-and- 
butter heart. Tuberculous infiltration of the myocardium was present in 6 ; the right auricle being involved in 3 , a generalized involvement in 2 , and involvement of the left auricle in the other. Active pulmonary tuberculosis was present in 8 , and in the remaining 8 the lungs were œdematous and congested. An infarct of the right basal lobe was present in 1 case. Here, as in the previous group, the root glands were tuberculous in every case. Pleural effusions were present in 10 ( 8 bilateral, 1 right-sided, and 1 left-sided). The liver was tuberculous in 7 and the kidneys in 7 . In 3 the spleen was tuberculous, and in 3 the intestine. Tuberculous mesenteric glands were present 4 times.

Treatment in our cases consisted of pericardial aspiration and the use of mercurial diuretics and digitalis. Later we began using the method of pneumopericardium. Two who had pericardial aspiration left hospital in a much improved condition. Pneumopericardium was carried out in two cases, one of whom died, whilst the other left hospital much improved.

Remarks.-Of the 19 patients reviewed above, 7 were seen by us in six months, and all were diagnosed clinically and confirmed biologically or by autopsy. They were usually admitted with the diagnosis of congestive cardiac failure, but a thorough examination and certain features, such as the febrile chart, the enlarged and pear-shaped area of cardiac dullness, the atypical distribution, and order of occurrence of the œdema, pointed the way to the correct diagnosis. The presence of a pericardial effusion is responsible for the picture of congestive heart failure as found in most cases. The effusion, as we have seen, may be either hæmorrhagic, serous, caseous, or a mixture of two types, the hæmorrhagic being the commonest. Loculation of the effusion due to intrapericardial adhesions may be present. Tuberculous infiltration of the myocardium was most commonly found in those cases with a caseous effusion. A feature of these cases was the absence of signs of pulmonary tuberculosis in most instances. Thus these cases fall chiefly into the clinically primary type of tuberculous pericarditis.

\section{(3) Cases passing through the Various Stages of Tuberculous PERICARDITIS}

The three cases in this group are important in helping to elucidate the course of tuberculous pericarditis. They demonstrate the passage of the condition through an acute stage to an effusion stage, and finally to the adherent stage.

Sex and Age.-All were males. Their ages were 26, 40, and 50 years respectively.

Histories.-The duration of their illness prior to admission was one, three, and eight weeks respectively. Breathlessness on exertion and œdema of the legs were complained of by one patient. All complained of cough, but none had had an hæmoptysis. One had precordial pain, and all abdominal pain.

Temperature and Pulse Rate.-Intermittent fever occurred in two, whilst the temperature was normal in the third.

The pulse rate was increased in all, and the respiratory rate was normal in all.

Clinical Signs.-Marked loss of weight was evident in two. Edema of the legs was present in one. 
Cardiovascular.-One had systolic apical retraction, and in two there appeared to be cardiac enlargement to the left. A pericardial friction rub was present in all three. This disappeared later in one. The heart sounds were normal.

Respiratory.-An impaired note with broncho-vesicular breath sounds was present in one at the right apex ; emphysema was found in one, and moist sounds were present in one throughout both lung fields.

Abdominal.-An enlarged and tender liver was found in one case, whilst the abdomen was generally tender in the other.

Autopsies.-A hæmorrhagic pericardial effusion loculated by intrapericardial adhesions was present in one. An adherent pericarditis was present in the other two and in one of them extrapericardial adhesions, a rare finding, were present between the heart and lungs. Tuberculous infiltration involving the right auricle existed in one. The root glands were tuberculous in all. Pulmonary tuberculosis was present in all, one having a tuberculous broncho-pneumonia with a terminal miliary spread ; in one, however, it was due to a direct infiltration from the root glands. The spleen and liver and the abdominal and cervical glands were tuberculous in one.

The outstanding feature in these cases was the presence of a pericardial friction rub. This is highly indicative of an acute stage of the condition. In the first particularly, one was able to follow the condition through the acute, effusion, and commencing adherent stages.

\section{Summary OF THE THREe Groups}

Incidence.-The coloured races are more commonly affected than the Europeans. Tuberculous pericarditis is more common in males than in females, 23 to 8 in this series. The difficulty in assessing the age incidence lies in the fact that the non-Europeans in this country rarely know their correct ages. The impression we have gained from this series is that the condition is commonest between the ages of 20 to 40 years and the ages given coincide with this ; cases, however, occurred from the age of 15 to the age of 76.

Pathology.-It is of considerable importance that in all the 28 cases with autopsies, the root glands were tuberculous. We think that these glands are the commonest source for the spread of tuberculous infection to the pericardium.

\section{The Manner in which Tuberculosis reaches the Pericardium}

There are three modes of spread :

(1) Spread by Direct Contact.-This is very rare and cannot be demonstrated in our series. It is, however, a possibility where tuberculous lungs or glands lie in direct contact with the pericardium. One would, then, expect to find the tuberculous process continuous with the adjacent focus, and spread throughout the parietal pericardial layer, but this is very rare and the probability is that even in these cases the spread takes place via the lymphatics.

(2) Spread by the Blood Stream.-This is a more common mode and usually 
occurs in the terminal miliary spread of a pulmonary tuberculosis. Miliary tuberculosis is a more common sequel of tuberculous pericarditis than vice versa. Not one case in this series resulted from a terminal miliary spread, whereas eleven cases of root gland and pericardial tuberculosis terminated in a miliary spread. When the infection is blood-borne it usually occurs before the condition can undergo further development.

(3) Spread by the Lymphatics.-This is undoubtedly the commonest mode. Most authors are agreed that a retrograde spread of infection takes place, but there has been some controversy as to the paths along which this occurs. Shore (1929) states that there is a close relationship between those glands receiving lymph drainage from the lungs and those from the heart, and describes a large mass of glandular tissue above the right lung root, the heart draining only into the lower part of this, which he calls the "caval gland of the heart." $\mathrm{He}$ further describes two lymph trunks draining the heart : (1) an anterior that drains chiefly the right ventricle and then empties into the medial members of a small group of glands lying in the mid-line of the body just below the left innominate vein ; and (2) a posterior that drains chiefly the left side of the heart and then empties into the caval gland of the heart. In addition to the above Shore describes the presence of small lymphatic glands interpolated between the points of formation and termination of the lymph-collecting trunks ; these are intra-pericardial in most instances.

From the above statements one deduces that the glands at the root of the lungs are the usual origin and source of spread of tuberculosis to the pericardium. The root glands, as we know, may be the first to become involved in cases of chest tuberculosis, and from here the infection may spread to the pericardium without the occurrence of any spread to the lungs. Pulmonary tuberculosis thus plays little part in the development of tuberculous pericarditis.

In corroboration of this we have seen that the root glands were tuberculous in every case in this series. On examination of the autopsy reports we found that the root glands on the right side, i.e. those including the caval gland of the heart, were most commonly involved. Pulmonary tuberculosis was present in six cases. Dr. Strachan states that he has seen the interpolated intrapericardial lymph glands involved by the tuberculous process in many cases of tuberculous pericarditis.

Myocardial tuberculosis was present in 10 of our cases-in 6 localized to the right auricle, in 3 generalized, and in 1 only chiefly localized to the left auricle. This suggests a lymphatic plexus or anastomosis in the region of the right auricle. In the same way, in most of the cases of tuberculous myocarditis reported by others the right auricle was most commonly involved.

\section{The Changes in the Pericardium}

These will be described in three stages.

(1) The Acute Stage.-The picture in this stage is one of an acute fibrinous pericarditis similar to that found in rheumatic fever. Its real nature may only 
be discovered on microscopical examination. Resolution, such as usually occurs in a rheumatic pericarditis, is rare.

(2) The Effusion Stage.-As the condition progresses, the pericardium, especially the parietal layer, becomes thicker, and this is accompanied by the outpouring of an exudate. The effusion is usually large, for example in one case over four pints of fluid. The fluid is most commonly hæmorrhagic in character, but may be straw-coloured or in some cases may consist of a caseous material similar to that found in tuberculous lungs or glands. It is at this stage that the clinical picture most typical of the condition is found.

(3) The Adherent Stage.-At this stage the effusion becomes smaller, thicker, grumous, or caseous. Adhesions develop between the pericardial layers resulting in loculation of the effusion. Loculation accompanied by roughened or shaggy pericardial surfaces probably only occurs in tuberculous pericarditis. Complete obliteration of the sac ultimately takes place as a result of the fluid becoming thicker and organized. At this stage the adhesions that form are usually intra-pericardial. Extra-pericardial adhesions were found in only three of our cases. This fact accounts for the rarity of systolic rib retraction. Adhesive pericarditis may occur primarily from fibroid tuberculosis without the first or second stages.

\section{SUMMARY AND CONCLUSIONS}

Pathological Survey.-Tuberculous root glands were present in all our cases. The tuberculous process was localized to the root glands and pericardium in 10 of the cases. In 6 the lungs, pericardium, and root glands were tuberculous. Terminal miliary tuberculosis occurred in 12 ; in 8 following primary root gland and pericardial tuberculosis and in 4 following primary lung, root gland, and pericardial tuberculosis. Pericarditis from a miliary spread was not seen. Pleural effusions were present in 15 cases, being bilateral in most ; 11 had either an acute pleurisy or pleural adhesions. Chronic congestion of the liver was present in most with either fibrous, fatty, or tuberculous changes.

Clinical Survey.-In spite of the short duration of the history, usually between three and six months, we believe the condition is usually insidious in its onset, and that these Bantu patients only began to notice symptoms when they became severe.

A history typical of congestive cardiac failure was generally given, especially in cases of the effusion type. Contrary to the statements of most authors, we found that there was usually complaint of cough. Hæmoptysis was rare, occurring in 5 cases. Abdominal pain, usually localized to the right hypochondrium, was complained of in 17 ; and 11 complained of chest pain, usually localized to the precordium. Edema of the legs was found in 11, breathlessness in 11, and 11 complained of loss of weight.

The temperature was raised in 22 , either a remittent or intermittent fever. The respiratory rate was increased in 20 , and the pulse rate in 29 ; in 3 there was a pulsus paradoxus. Auricular fibrillation and extrasystoles were each seen 
once. A febrile chart, rapid pulse, and breathlessness were found chiefly with pericardial effusions.

Most were poorly nourished, and 15 had an emaciated appearance. Edema was present in the legs of 12 and in 3 it was generalized.

Cardiac murmurs were only present in 2 , and a pericardial rub in 3 . The heart sounds were usually muffled.

Only 9 had signs of pulmonary tuberculosis ; it was of the primary clinical type of Riesman (1901). Moist sounds at the bases were found commonly.

The liver was enlarged, and usually exquisitely tender, in 12 ; and 8 had ascites.

The effusion stage is, as we have shown, the easiest one in which to make a clinical diagnosis, the enlarged pear-shaped area of cardiac dullness with an obtuse cardio-hepatic angle being the outstanding sign.

\section{Prognosis}

The prognosis is bad. Death usually occurs in one to six months after the onset of symptoms. The condition, however, is not always fatal, as shown by the fact that people dying of other causes have been found to have a healed tuberculous pericarditis. We believe that pericardial aspiration and pneumopericardium improve the prognosis as far as the time of survival is concerned. This belief is substantiated by the fact that in the only cases which left hospital the pericardium had been aspirated; in one case pneumopericardium had been performed in addition.

The cause of death is either cardiac failure or, more commonly, miliary tuberculosis ; this is common in tuberculous pericarditis and may be due to the presence of tubercle bacilli in the fluid and tissues surrounding the heart. The probable mode of entry of these organisms into the blood stream, particularly in cases with intra-pericardial caseation, is by the rupture of a tuberculous follicle into a vein.

\section{Diagnosis}

The two main considerations are the diagnosis of pericardial disease and the establishment of its tuberculous nature.

The main points establishing the former are as follows :

(a) In the Acute Stage.-The presence of a pericardial rub.

(b) In the Effusion Stage.-(1) The absence of a visible or palpable apex beat. (2) The enlarged pear-shaped area of cardiac dullness. (3) The presence of Rotch's angle. (4) The typical radiographic picture of pericardial effusion. (5) Pericardial aspiration.

(c) In the Adherent Stage.-(1) Evidence of cardiac incompetence. (2) Evidence of venous engorgement. The condition is most difficult to diagnose at this stage, and systolic retraction of the ribs has been seen only once. 
The following are in favour of a pericarditis being tuberculous :

(1) A patient suffering from a chronic wasting disease.

(2) Fever, intermittent or remittent, with frequent inverse manifestations.

(3) Pulmonary tuberculosis or active tubercle elsewhere (almost conclusive).

(4) Congestive cardiac failure, with a febrile chart and bilateral pleural effusions, without organic cardiac murmurs or evidence of hypertensive disease or coronary thrombosis. Also swelling of the face without kidney damage.

(5) Hæmorrhagic pericardial fluid.

(6) Radiography and probably kymography after pneumopericardium, showing the small heart and thickened pericardial wall.

(7) The finding of tubercle bacilli in the pericardial fluid.

We desire to thank the honorary staff of the Johannesburg General Hospital for permission to use their cases, Drs. Weinbrein and Meyer for their kymographs and reports, and Dr. A. S. Strachan for his autopsy reports and for his scrutiny and advice in regard to the pathological section.

\section{REFERENCES}

Bellet, S., McMillan, T. M., and Gouley, B. A. (1933). Med. Clin. N. Amer., 18, 201. Burrel, L. S. T., Hare, D. C., and Ross, J. M. (1929). Lancet, 2, 1303.

Keefer, C. S. (1937). Ann. intern. Med., 10, 1085.

Mackay, N. R. (1937). N. Zealand med. J., 36, 41.

Rawls, W. B. (1925). Amer. J. med. Sci., 199, 815.

Riesman, J. (1901). Amer. J. med. Sci., 122, 6.

Shore, L. R. (1929). J. Anat., Lond., 63, 291.

Smith, H. L., and Willius, F. A. (1932). Arch. intern. Med., 50, 121.

Thomas, G. W. (1932). Amer. Heart J., 7, 771.

Thompson, W. P. (1933). J. Amer. med. Ass., 100, 642.

Waller, W. E. (1923). Lancet, 2, 278.

Wells, H. G. (1902). Amer. J. med. Sci., 123, 241.

White, P. D. (1937). Diseases of Heart.

Wilson, F. N., and Hills, C. R. (1922). Med. Clin. N. Amer., 6, 1210. 